\title{
Development of automated neighborhood pattern sensitive fault syndrome generator for SRAM
}

\begin{abstract}
With the increasing complexity of memory devices, fault diagnosis is becoming as important as fault detection. Fault diagnosis is to locate and identify type of fault. One of the memory faults is Neighborhood Pattern Sensitive Faults (NPSF) which is one of the faults that are hard to test due to higher number of cells to be tested at one time. To improve the process of analyzing NPSF detection and to generate the fault syndrome for NPSF diagnosis, an Automated NPSF Syndrome Generator (ANPSFSG) is developed. A proven March algorithms are used in this generator to verify the efficiency of this generator by producing the fault coverage and diagnostic resolution. A user-friendly Graphical User Interface (GUI) of ANPSFSG is also developed by using Microsoft Visual Basic software to load the algorithm under test and display the results.
\end{abstract}

Keyword: ANPSFSG; GUI; March algorithm; Memory diagnosis; Memory fault syndrome; Memory testing; NPSF; SRAM 